\title{
Time Constant Estimation of Asymptotically Stable Linear System by Lyapunov Function
}

\author{
Shengguo Zhang ${ }^{1, ~ a, ~ S h u o ~ Z h a n g ~}{ }^{2, ~ b}$, Wanchun Liu' ${ }^{1, c}$, Jingtao Huang ${ }^{1, ~ d, ~ a n d ~}$ \\ Guoheng Zhang ${ }^{1, e}$ \\ ${ }^{1}$ School of Electrical Engineering, Northwest University for Nationalities, Lanzhou, 730030, China \\ ${ }^{2}$ School of Automation, Hangzhou Dianzi University, Hangzhou, 310018, China \\ azhangshengguo@tsinghua.org.cn, b407459957@qq.com, ${ }^{c} 1054828395 @ q q . c o m$, \\ d394474489@qq.com, ${ }^{\mathrm{e}}$ 123163154@qq.com
}

Keywords: Energy attenuation rate, Initial state response, Lyapunov function, Time constant. Abstract: This paper aims at estimating the time constant of the asymptotically stable control system. The energy varying rate of the system states is defined based on Lyapunov function and its differential function. The minimum energy attenuation rate is defined and solved by the function condition extreme method. The system time constant is estimated to be double of the multiplicative inverse of the minimum energy attenuation rate by taking into account of that the system energy is one order higher than the system state. Application example about a third order control system indicates that the solved time constant is just the response time of the initial states and yet it is irrelevant to the initial states of the system. So this time constant can serve as the estimation value of the response time and this estimated time constant can be used to evaluate the rapidity performance for the asymptotically stable control system.

\section{Introduction}

The time constant is an important index to evaluate the dynamic performances of the asymptotically stable control system. It manifests the response speed to the initial condition or the reference input of the control system. Usually, the time constant is derived from the unit step response of the control system and it is assessed by the indexes such as delay time, rising time, peak time, and settle time etc. However, different control systems have different time constants. It is difficult to obtain this time constant by the analytical method especially for the higher order control system [1].

From Lyapunov stability theory [2,3], it is noticed that Lyapunov function is an energy function in essence and its differential function about the time represents the varying rate of the energy of the system states. When the energy of the system states is exhausted asymptotically, the system must tend to its equilibrium states and thus the system is deemed to be asymptotically stable [4]. So in this paper, Lyapunov function and its differential function are used to estimate the time constant of the control system. An energy varying rate function, which results from Lyapunov function and its differential function, is defined first. Then the minimum energy attenuation rate is defined and solved by the function condition extreme in which Lyapunov equation is used. And then the multiplicative inverse of the minimum energy attenuation rate is calculated and its twofold is defined to be the time constant of the free response of the control system.

\section{Time Constant Estimation Based on Lyapunov Function and Its Differential Function}

Definition of the Minimum Energy Attenuation Rate. A linear time-invariant (LTI) control system can be represented by state space model as:

$$
\left\{\begin{array}{l}
\boldsymbol{A} x+\boldsymbol{B} u \\
y=\boldsymbol{C} x
\end{array},\right.
$$


where $x$ is state vector, $u$ is control input vector, and $y$ is output vector. $\boldsymbol{A}, \boldsymbol{B}$ and $\boldsymbol{C}$ are state matrix, input matrix and output matrix with appropriate dimensions, respectively. For this control system, its energy can be represented by Lyapunov function, $V(x)$, which is a positive definite quadratic function:

$$
V(x)=x^{\mathrm{T}} \boldsymbol{P} x,
$$

where $\boldsymbol{P}$ is an arbitrary symmetrical positive definite matrix. And its differential function $\mathcal{L}_{x}$ ) can be represented as a negative definite quadratic function:

$$
\mathscr{Q}(x)=\frac{\mathrm{d} V(x)}{\mathrm{d} t}=-x^{\mathrm{T}} \boldsymbol{Q} x,
$$

where $Q$ is also a symmetrical positive definite matrix.

In accordance with above definitions, $V(x)$ can scale the energy distance from the current system state $x$ to the system equlibrium state $x_{e}$, and $\alpha_{x}$ ) can scale the energy varying speed from the current system state $x$ to the system equlibrium state $x_{e}$. So an index $\gamma$, which can scale the system energy varying rate, is defined as:

$$
\gamma=-\frac{1 \&(x)}{V(x)}
$$

This energy varying rate is also the function of system state $x$. If the system is asymptotically stable, $V(x)$ is positive and $\delta_{(x)}$ is necessarily negative, and therefore $\gamma$ is positive necessarily. The greater $\gamma$ is, the more quickly $V(x)$ decreases, and the more quickly the system state $x$ tends to the equilibrium state $x_{e}$. Nevertheless, due to the nonuniqueness of Lyapunov function, it is difficult to get the energy varying rate $\gamma$ directly. For this reason, defining the minimum of $\gamma$ and naming it the minimum energy attenuation rate $\gamma_{\min }$ :

$$
\gamma_{\min }=\min \left[-\frac{V(x)}{V(x)}\right],
$$

this is a constant for the asymptotically stable control system and it indicates the minimum decay speed of the system energy.

Solving the Minimum Energy Attenuation Rate. According to the definitions Eq. 2 and Eq. 3, Eq. 5 can be derived as:

$$
\gamma_{\text {min }}=\min \left[-\frac{V(x)}{V(x)}\right]=\min \left[\frac{x^{\mathrm{T}} \boldsymbol{Q} x}{x^{\mathrm{T}} \boldsymbol{P} x}\right],
$$

where $\boldsymbol{P}$ and $\boldsymbol{Q}$ meet Lyapunov equation because of the asymptotical stability of the control system:

$$
\boldsymbol{A}^{\mathrm{T}} \boldsymbol{P}+\boldsymbol{P A}=-\boldsymbol{Q}
$$

In order to solve the minimum energy attenuation rate $\gamma_{\min }$, normalizing the Lyapunov function $V(x)$, i.e. let $x^{\mathrm{T}} \boldsymbol{P} x=1$, then there yields:

$$
\boldsymbol{\gamma}_{\min }=\min \left[\frac{x^{\mathrm{T}} \boldsymbol{Q} x}{x^{\mathrm{T}} \boldsymbol{P} x}\right]=\min _{x}\left[x^{\mathrm{T}} \boldsymbol{Q} x, x^{\mathrm{T}} \boldsymbol{P} x=1\right],
$$

it is a function extreme problem with equality constraint condition. And then by setting up a Hamilton function:

$$
H(x, \mu)=x^{\mathrm{T}} \boldsymbol{Q} x+\mu\left[x^{\mathrm{T}} \boldsymbol{P} x-1\right]
$$


the function extreme problem with equality constraint condition is turned to be a function extreme problem without constraint condition. Solving this function extreme problem, there gets:

$$
\gamma_{\min }=\lambda_{\min }\left(\boldsymbol{Q P} \boldsymbol{P}^{-1}\right)
$$

where $\lambda_{\text {min }}\left(\boldsymbol{Q P} \boldsymbol{P}^{-1}\right)$ denotes the minimum eigenvalue of matrix $\boldsymbol{Q P}{ }^{-1}$.

Time Constant Estimation. In essence, Lyapunov function $V(x)$ is an energy function. In the viewpoint of physics, energy $V(x)$ is one order higher than the system state $x$. So the decaying speed of $V(x)$ is quicker than the decaying speed of the system state $x$. Under this consideration, the system time constant $\mathrm{T}_{\max }$, which is the system state decaying time of free response, is estimated to be the two times of the multiplicative inverse of the minimum energy attenuation rate $\gamma_{\min }$ :

$$
\mathrm{T}_{\max }=\frac{2}{\gamma_{\min }}
$$

\section{Application Example}

There is a third order control system and its state equation is as following:

$$
\&=\left[\begin{array}{ccc}
0 & 1 & 0 \\
0 & -2 & 1 \\
-3 & 0 & -1
\end{array}\right] x+\left[\begin{array}{l}
0 \\
0 \\
3
\end{array}\right] u
$$

Given $Q$ to be a symmetrical positive definite matrix, e.g. three-dimension unit matrix:

$$
\boldsymbol{Q}=\left[\begin{array}{lll}
1 & 0 & 0 \\
0 & 1 & 0 \\
0 & 0 & 1
\end{array}\right]
$$

then solving Lyapunov equation:

$$
\left[\begin{array}{ccc}
0 & 0 & -3 \\
1 & -2 & 0 \\
0 & 1 & -1
\end{array}\right] \boldsymbol{P}+\boldsymbol{P}\left[\begin{array}{ccc}
0 & 1 & 0 \\
0 & -2 & 1 \\
-3 & 0 & -1
\end{array}\right]=-\left[\begin{array}{lll}
1 & 0 & 0 \\
0 & 1 & 0 \\
0 & 0 & 1
\end{array}\right],
$$

the symmetrical positive definite matrix $\boldsymbol{P}$ is derived:

$$
\boldsymbol{P}=\left[\begin{array}{ccc}
10.8333 & 4.1667 & 0.1667 \\
4.1667 & 2.3333 & 0.8333 \\
0.1667 & 0.8333 & 1.3333
\end{array}\right],
$$

then the eigenvalues of matrix $\boldsymbol{Q P} \boldsymbol{P}^{-1}$ can be solved:

$$
\lambda_{1}=0.0797, \lambda_{2}=0.5687, \lambda_{3}=5.3516,
$$

thus, the minimum energy attenuation rate can be derived:

$$
\gamma_{\min }=\min \left(\lambda_{1}, \lambda_{2}, \lambda_{3}\right)=0.0797,
$$

and the system time constant $\mathrm{T}_{\max }$ can be calculated to be:

$$
\mathrm{T}_{\max }=\frac{2}{\gamma_{\min }}=25.1096 .
$$


If there is given another symmetrical positive definite matrix $\boldsymbol{Q}$, the solved symmetrical positive definite matrix $\boldsymbol{P}$ will also be changed. Anyway, the minimum eigenvalue of matrix $\boldsymbol{Q} \boldsymbol{P}^{-1}$ is nearly unchangeable, and so the system time constant $\mathrm{T}_{\max }$ is nearly unchangeable.

In order to verify the system time constant $\mathrm{T}_{\max }$, given arbitrary initial states, e.g. $x_{\mathrm{i} 1}=1, x_{\mathrm{i} 2}=0$, and $x_{\mathrm{i} 3}=1$, the free response of the system, i.e. the initial state responses of the system are drawn as Fig. 1 is shown. From this figure, it can be seen that three state responses are all getting into the range from -0.02 to 0.02 within 25.1096 seconds. Given another initial states, the response time is approximatively unchangeable. This result indicates that above estimated time constant is correct.

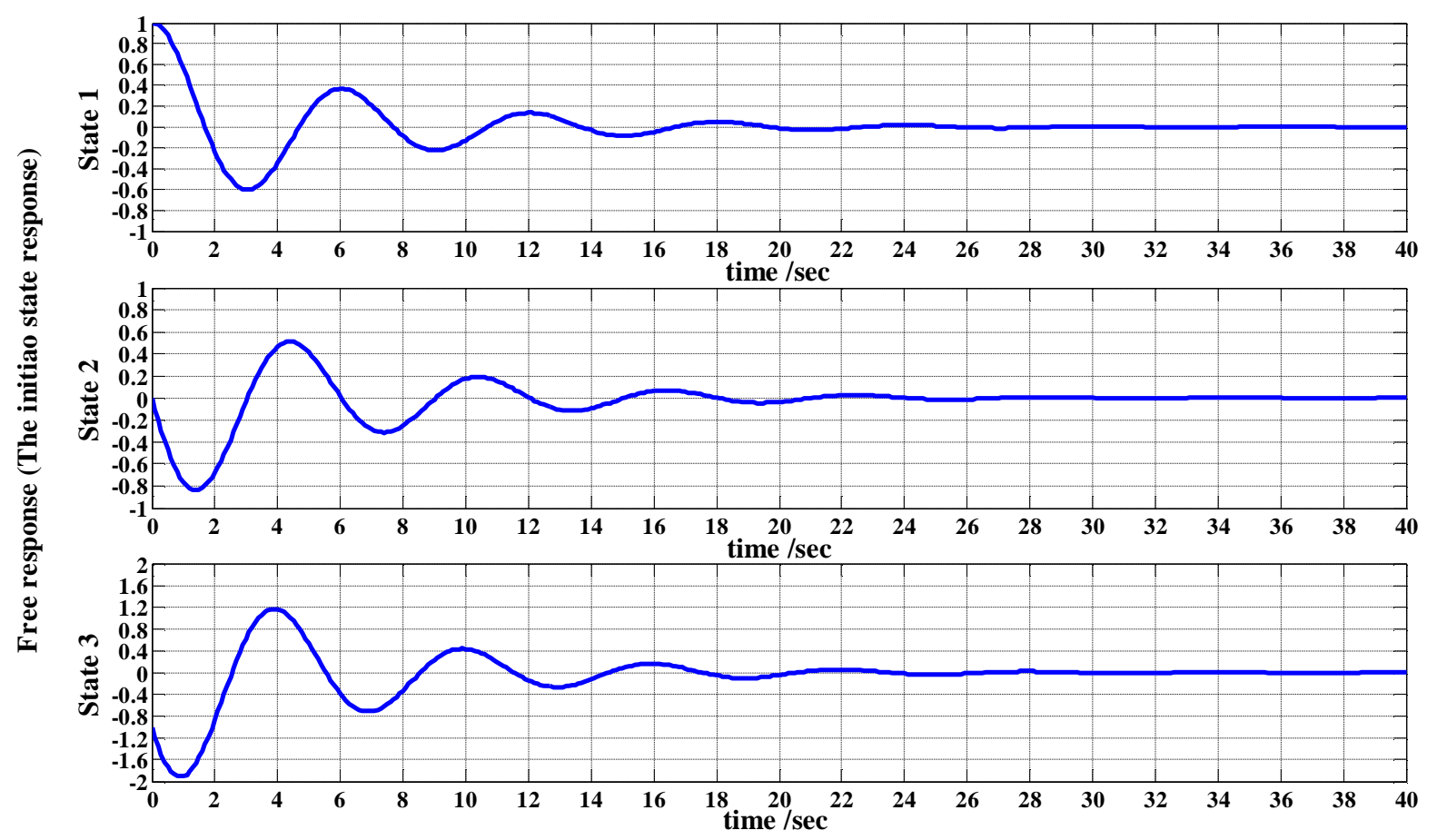

Fig. 1 Free response of the system (The initial state responses)

\section{Conclusion}

The time constant of the asymptotically stable control system is estimated on the basis of Lyapunov function and its differential function. By Lyapunov function and its differential function, the system energy varying rate is defined and the minimum of this energy varying rate is defined as the minimum energy attenuation rate. By function condition extreme method, the minimum energy attenuation rate is solved and it is the minimum eigenvalue of a matrix which is dependent upon Lyapunov equation. And then the time constant, which is the decaying time of the initial states of the system, is estimated as double of the multiplicative inverse of the minimum energy attenuation rate under the consideration that the system energy is one order higher than the system state.

\section{Acknowledgements}

This study was supported by the National Natural Science Foundation of China (51465053) and the Fundamental Research Funds for the Central Universities (31920140082).

\section{References}

[1] Shousong Hu, Automatic Control Principle, fifth ed., Science Press, Beijing 2007.

[2] A. Bacciotti and L. Rosier, Lyapunov Functions and Stability in Control Theory, Springer-Verlag 2005. 
[3] M. Sassano and A. Astolfi, Dynamic Lyapunov Functions: Properties and Applications, 2012 American Control Conference, 2012, 2571-2576.

[4] Li Yu, Modern Control Theory, Tsinghua University Press, Beijing 2007. 